\title{
Naive Haftpflichtklage oder tragischer Regulierungsfehler?
}

\section{Konstantin Beck}

Prof. Dr. oec., Leiter CSS Institut für empirische Gesundheitsökonomie Luzern und Universität Zürich

\author{
Beim Produkt Yasmin ${ }^{\circledR}$ ist die Risikodeklaration sowohl gegenüber den Patientin- \\ nen als auch gegenüber den Ärzten irreführend und in zentralen Punkten unvoll- \\ ständig. Es ist nicht auszuschliessen, dass die Kosten der Nebenwirkungen höher \\ als die erwirtschafteten Gewinne sind. Die Kostenfolgen der Nebenwirkungen \\ muss aber die Allgemeinheit tragen.
}

\section{Céline contra Yasmin}

Beim Fall Céline contra Yasmin ging es um eine junge Frau, die das hormonelle Kontrazeptivum Yasmin einnahm und nach zwei Monaten, im März 2008, ein schweres venöses thromboembolisches Ereignis (VTE) erlitt. Infolgedessen ist sie heute spastisch gelähmt und schwer invalid. Die Betroffene führte das VTE kausal auf die Einnahme der Pille zurück und führte eine Haftungsklage gegen den Hersteller (Bayer Schweiz). Hormonelle Kontrazeptiva erhöhen in der Tat die Wahrscheinlichkeit von Thrombosen und ein VTE führt in $20 \%$ der Fälle zu Invalidisierung und in 1-2\% der Fälle zum Tod [1]. Enthält das Medikament Drospirenon, so verdoppelt sich das Thromboserisiko im Vergleich mit älteren Pillen mit Levonorgestrel [2]. Ge-
Lesen Sie hierzu auch den
Kommentar von Swissmedic im Anschluss an diesen

Beitrag. klagt wurde u.a., weil es Bayer unterlassen hatte, in der (zum damaligen Zeitpunkt gültigen) Patienteninformation auf das doppelt so hohe Risiko hinzuweisen (vgl. [3]). Die Klage wurde jedoch am 5. Januar 2015 vom Bundesgericht in dritter Instanz abgewiesen. Das Gericht argumentierte, rezeptpflichtige Medikamente müssten vom Arzt verschrieben werden. Das gehe einher mit einer Aufklärungspflicht des verschreibenden Arztes. Es sei daher unerheblich, dass das erhöhte Risiko nur in der Fachinformation des Arztes und nicht auch in der Patienteninformation angesprochen werde [4].

Das Gericht nimmt somit an, die Summe der Information von Fach- und Patienteninformation sei korrekt und vollständig. Im Folgenden soll diese Annahme überprüft werden. Zudem zeigen wir abschliessend, wie das Risiko bei Verhütungsmitteln aussagekräftiger in Franken und Rappen dargestellt werden könnte.

\section{Wird das Risiko von Bayer verzerrt dargestellt?}

Ausgehend vom oben dargestellten Rechtsstreit bezieht sich unsere Risikoanalyse auf zwei Zeitpunkte: Einerseits untersuchen wir die Patienten- und die Fachinformation (von 2004 respektive 2007), die Céline und ihrem Arzt beim Kauf bzw. bei der Verschreibung als Entscheidungsgrundlagen zur Verfügung standen [3, 5], andererseits untersuchen wir die heute vorliegenden Informationen [2, 6].

Tabelle 1 stellt die Risiken der VTE pro 10000 Frauenjahren dar, wie sie in den entsprechenden Informationen und im Expertenbrief der Schweizerischen Gesellschaft für Gynäkologie und Geburtshilfe (SGGG) aufgeführt sind. 
Tabelle 1: Inzidenz venöser Thromboembolien (VTE) pro 10000 Frauenjahre.

\begin{tabular}{|c|c|c|c|c|}
\hline Quellen & $\begin{array}{l}\text { Nicht-Anwenderinnen } \\
\text { oraler Kontrazeptiva }\end{array}$ & Schwangere & $\begin{array}{l}\text { Anwenderinnen komb. } \\
\text { Kontrazeptiva }\end{array}$ & $\begin{array}{l}\text { Anwenderinnen } \\
\text { komb. Kontrazeptiva } \\
\text { mit Drospirenon }\end{array}$ \\
\hline Patienteninformation 2004 [3] & Keine Zahlenangabe & Keine Zahlenangabe & $\begin{array}{l}\text { Risiko leicht erhöht, } \\
\text { geringer als bei } \\
\text { Schwangerschaft }\end{array}$ & Keine Zahlenangabe \\
\hline Fachinformation 2007 [5] & $0,5-3$ & 6 & 4 & $\begin{array}{l}\text { Allgemeiner } \\
\text { Warnhinweis }\end{array}$ \\
\hline SGGG 2013 [1] & $\begin{array}{l}\text { Alter } 15-35: 1-2 \\
35-44: 3-5\end{array}$ & $8-30$ & $5,5-10$ & $\begin{array}{l}\text { Verdoppelung } \\
\text { bezogen worauf? }\end{array}$ \\
\hline
\end{tabular}

Das Positive vorweg: In sämtlichen genannten Dokumenten wird explizit auf das Risiko von Gerinnselbildungen in Venen und Arterien hingewiesen und die damit verbundenen Gefahren (Lungenembolie, Schlaganfall oder Herzinfarkt) werden explizit aufgezählt und als "teilweise schwere Gesundheitsschäden" eingestuft (Zitat aus [3]). Weitere diskutierte Nebenwirkungen interessieren im vorliegenden Zusammenhang nicht.

Wir untersuchen in den folgenden fünf Punkten, wie Bayer das Nebenwirkungs-Risiko darstellt.

\section{Wird das Risiko abgeschwächt?}

Bayer spricht von einem leicht erhöhten Risiko für Gerinnselbildungen in Venen und Arterien, die zu teilweise schweren Gesundheitsschäden führen können $[3,5]$. Eine Umfrage bei 43 Bachelor-Studenten der Universität Zürich ergab, dass $93 \%$ unter «leicht erhöht» einen Anstieg um nicht mehr als das Doppelte, 86\% sogar einen Anstieg um weniger als das Doppelte verstehen [7]. Die Formulierung von Bayer entspricht weder dem, was umgangssprachlich unter einem «leicht erhöhten Risiko» verstanden wird, noch dem, was in Tabelle 1, Zeile 2 ersichtlich ist. Dort steigt das Risiko von 0,5 Fällen pro 10000 Frauenjahre auf 4 Fälle, was einem Anstieg um den Faktor 8 (!) entspricht. Gigerenzer empfiehlt verschiedentlich, Risiken als Numbers needed to treat auszudrücken (vgl. [8]). Kommt bei den Nichtanwenderinnen 1 Betroffene auf 20000 Frauen, so ist es bei Anwendung von Yasmin 1 Betroffene auf 2500 Frauen, was einer recht erheblichen Veränderung des Risikos entspricht (ohne dass der Warnhinweis in [5] als zusätzliches Risiko hineingerechnet worden wäre).

Immerhin wurde in den jüngeren Publikationen das Adjektiv «leicht» gestrichen. Die Risikoinformation bleibt jedoch eingebettet in abschwächende Aussagen wie «Solche Ereignisse können unabhängig davon auftreten, ob Sie die Pille anwenden oder nicht» [6].

\section{Wird das Risiko unvollständig ausgewiesen?}

Die von Bayer ausgewiesenen Zahlenwerte beziehen sich alle ausschliesslich auf venöse Thrombosen. James weist auf Publikationen von 2005 und 2006 hin, die für Schwangere nachweisen, dass auf vier venöse eine arterielle Thrombose komme [9]. Gemäss SGGG ([1]) ist das arterielle Risiko bei kombinierten Präparaten höher als das natürliche. Es ist einzig unklar, ob Drospirenon das arterielle Risiko darüber hinaus zusätzlich erhöht. Beide Quellen stützen die These, dass alle in Tab. 1 aufgeführten Risiken zu gering ausgewiesen werden. Zudem wird verbal auf ein höheres Risiko bei Neuanwenderinnen hingewiesen. Die Höhe dieses Einsteiger-Risikos wird ebenfalls nicht quantifiziert.

\section{Ist der Risikovergleich verfälscht?}

Eine eigentümliche Entwicklung des Risikovergleichs (immer im Vergleich zur SGGG) setzt 2012 ein. Bayer hebt dort das Risiko von Yasmin ${ }^{\circledR}$ um 200\% bis $250 \%$ an, was bei neuen Medikamenten nicht aussergewöhnlich ist. Erstaunlich ist hingegen der starke Anstieg beim Risiko von Nicht-Anwenderinnen und Schwangeren. Diese seit Jahren bekannten Risiken steigen um bis zu $800 \%$ respektive $500 \%$. Ein Blick in die von der SGGG zitierte Literatur stützt die Risikoangaben von Bayer nicht. Vergleichen wir Zeile 3 mit Zeile 4 (Tab.1), so liegt die Mehrheit der SGGG-Angaben zum natürlichen Risiko unter den Angaben von Bayer und keine einzige übertrifft die Angaben von Bayer. Indem Bayer das Referenzrisiko sehr hoch ansetzt, schwächt es das zusätzliche Risiko kombinierter Kontrazeptiva deutlich ab.

\section{Wird das relevante Risiko verschwiegen?}

Nun interessiert hier nicht so sehr das Risiko anderer Kontrazeptiva (Tab. 1, Spalte 3) sondern das spezifische Risiko von Yasmin ${ }^{\circledR}$ (Tab. 1, Spalte 4). Kern der Argu- 


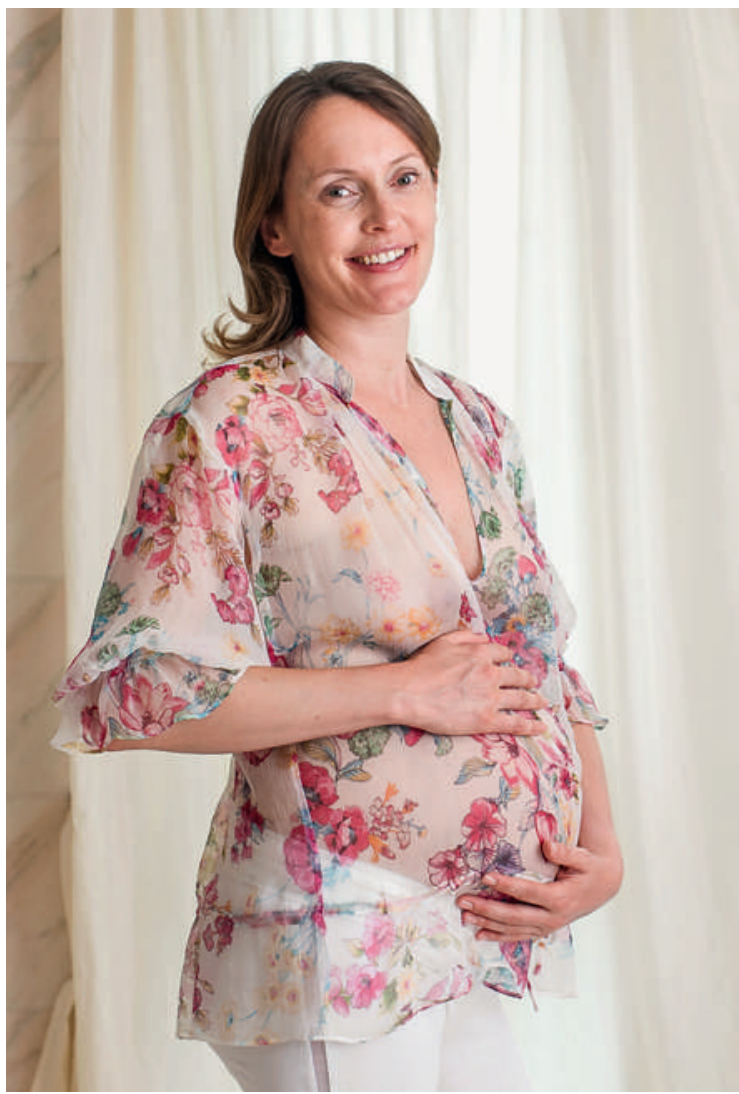

Die gesichtete Literatur widerspricht der Aussage, eine Schwangerschaft erhöhe das Thromboserisiko für junge Frauen mehr als die Einnahme von Yasmin ${ }^{\circledR}$.

mentation zu den VTE-Inzidenzen in der Fach- und Patienteninformation 2012 [2, 6] ist ausgerechnet eine vom Hersteller von Yasmin ${ }^{\circledR}$ finanzierte EURAS-Studie, welche keinen signifikanten Unterschied im Risiko von Drospirenon verglichen mit anderen Wirkstoffen nachweist (was im Informationsschreiben allerdings unklar bleibt). Die Studie wurde aus methodischen Gründen heftig kritisiert (vgl. [10]). So ist das Vertrauensintervall so breit, dass auch ein $80 \%$ höheres VTE-Risiko von Drospirenon als nicht signifikant angesehen wird (ebenda). In beiden Informationsschreiben von 2012 wird eine doppelt so hohe VTE-Inzidenz für Anwenderinnen kombinierter Kontrazeptiva angegeben (8-10 VTE pro 10000 Frauenjahre verglichen mit 4-5 VTE bei Nichtanwenderinnen). Die Patienteninformation (nicht aber die Fachinformation) schliesst einen dreifachen Anstieg nicht aus (was 12-15 VTE entspräche).

Weiter unten wird dann doch auf eine Verdoppelung der Inzidenz bei Yasmin ${ }^{\circledR}$ im Vergleich zu älteren Kontrazeptiva hingewiesen. Allerdings gestützt auf andere Studien, so dass nicht einfach 8-10 VTE (respektive 12-15 VTE) verdoppelt werden können. Somit hängt die Verdoppelungs-Aussage in der Luft, weil offen bleibt, auf welche Inzidenz sie sich konkret bezieht. Die Inzi- denz muss «irgendwo» oberhalb von 8-10 respektive 12-15 VTE zu liegen kommen. Obwohl Bayer in den Fassungen von 2012 mehr Zahlenangaben macht als früher, kann die eigentlich interessierende Grösse, die VTE-Inzidenz für Yasmin ${ }^{\circledR}$ nicht gefunden werden.

\section{Wie riskant ist eine Schwangerschaft wirklich?}

Die Bedeutung des Thromboserisikos bei Schwangerschaften darf nicht unterschätzt werden. Mit dem Thromboserisiko bei Schwangeren setzt der Verkäufer von Yasmin ${ }^{\circledR}$ bei einem für viele Frauen unvermeidbaren Risiko an. Ist dieses Risiko höher als das des Verhütungsmittels, dann fällt der Entscheid zu Gunsten von Yasmin ${ }^{\circledR}$ relativ leicht.

2012 setzt Bayer das Risiko für junge Schwangere sehr hoch bei 20 Fällen an. Das übersteigt den Yasmin ${ }^{\circledR}$ Wert von 8 (ev. 12) Fällen pro 10000 . Gerade bei den jungen Schwangeren ist die Differenz zur SGGG besonders gross. Die gesichtete Literatur spricht von lediglich 7,6 Fällen pro 10000 ([11], S. 2025) und kann damit die Aussage, eine Schwangerschaft sei gefährlicher als die Einnahme von Yasmin, für junge Frauen nicht stützen.

\section{Gewinne zu Gunsten von Bayer - Kosten zu Lasten der Allgemeinheit?}

Es stellt sich nun aber die Frage, ob der Arzt in die Pflicht genommen werden darf, wie es das Bundesgericht tut, wenn er selber Opfer unvollständiger und höchst problematischer Risikoangaben ist, die zumal von Swissmedic amtlich abgenommen worden sind. Liegt die Haftung beim Arzt, so spürt das die Ärzteschaft in Form steigender Haftpflichtprämien. Das wäre umso stossender, als dass Bayer schon jetzt zwar

\section{Indem Bayer das Referenzrisiko sehr hoch} ansetzt, schwächt es das zusätzliche Risiko kombinierter Kontrazeptiva deutlich ab.

den Gewinn des Produkts einstreicht, die Kostenfolgen der Nebenwirkungen aber der Allgemeinheit (genauer: den Sozialversicherungen) aufbürdet. Eine vollständige Risikobeurteilung sollte daher immer auch die Kosten berücksichtigen.

Beck [7] legt eine detaillierte Berechnung der Kosten der schweren, invalidisierenden VTE pro Jahrespackung Yasmin $^{\circledR}$ vor. Er kann sich allerdings nur auf ein kleines Kollektiv von 5 VTE-Patientinnen abstützen. Deren Durchschnittskosten betragen 2,25 Mio. Franken. Dabei fehlen noch die Kosten der IV und die Vollkosten des 
Pflegeheims (Angaben, die in der verwendeten Krankenversicherungsstatistik nicht enthalten sind). Zudem erwartet man im eingangs besprochenen Fall mittlerweile Gesamtkosten von 4,6 Mio. Franken.

VTE treten auch natürlicherweise auf. Dem Medikament darf daher nicht das gesamte Risiko sondern nur das zusätzliche Risiko nach Abzug der natürlichen Inzidenz angerechnet werden. In Abzug gebracht wird das Risiko bei Schwangerschaft (hergeleitet aus der Anzahl Geburten korrigiert um Mehrlingsgeburten [12], der Anzahl Abtreibungen [13] und der Anzahl Spontanaborte) und die natürliche Inzidenz bei Nicht-Schwangeren. Gerechnet wurde mit den eher zu hohen natürlichen Inzidenzen, die Bayer 2012 ausweist (Tab. 1, Zeile 3). Das Resultat wird wie gesagt eingeschränkt auf die 20\% VTE mit invalidisierenden Folgen (gemäss [1]). Vollkommen unberücksichtigt blieben die arteriellen Thrombosen sowie der Risikoanstieg, der auf den Wirkstoff in Yasmin zurückgeführt werden kann (!). Der Berechnung zugrunde liegt die Annahme einer Verdoppelung respektive einer Verdreifachung der natürlichen VTE-Inzidenz (gemäss [6] und [2]). Alle diese Annahmen führen zu einer Unterschätzung der Risikokosten von Yasmin.

Während der Verkaufspreis von Yasmin ${ }^{\circledR}$ bei 230 CHF pro Jahrespackung liegt ${ }^{1}$, beläuft sich der Risikozuschlag auf 149 CHF bis 351 CHF pro Jahr. Das sind $65 \%$ bis 153\% des Verkaufspreises. Diese Werte stellen die Untergrenze der Risikokosten dar, wenn das Kontrazeptivum die natürliche Inzidenz lediglich verdoppelt oder verdreifacht.

\section{Fazit}

Während eine Jahrespackung Yasmin ${ }^{\circledR} 230$ CHF kostet, verursacht sie Risikokosten zwischen 149 CHF und 351 CHF. Diese Werte stellen eine Untergrenze dar. Substitute ohne Drospirenon verursachen sehr wahrscheinlich geringere Risikokosten. Auf Grund der nach wie vor ungenügenden Risikoinformation in Packungsbeilage und Fachinformation lassen sich die Unterschiede zwischen Produkten mit und ohne Drospirenon nicht berechnen. Unsere Berechnung zeigt, dass die Allgemeinheit den Kauf jeder Packung Yasmin ${ }^{\circledR}$ mit 149 CHF bis 351 CHF quersubventioniert. Es bleibt zu vermuten, dass der ganze von Bayer erwirtschaftete Erlös die verursachten Schäden nicht zu decken vermag.

Beim Produkt Yasmin ${ }^{\circledR}$ ist die Risikodeklaration sowohl gegenüber den Patientinnen als auch gegenüber den Ärzten irreführend und in zentralen Punkten unvollständig. Demgegenüber ist nicht auszuschliessen, dass die Kosten der Nebenwirkungen höher als die erwirtschafteten Gewinne sind. Es stellt sich also die Frage, warum Swissmedic ein so hochproblematisches Produkt nicht vom Markt nahm.

\section{Danksagung}

Der Autor dankt der CSS Versicherung AG für die Unterstützung dieser Analyse. Die hier geäusserte Meinung ist die des Autors und deckt sich nicht zwingend mit der Meinung der CSS-Gruppe.

\section{Bildnachweis}

MEV Verlag GmbH, Germany

\section{Literatur}

1 Merki-Feld G, Bitzer, Seydoux J, Birkhäuser M. Expertenbrief (No 35) zum Thromboembolierisiko unter hormonaler Kontrazeption. SGGG, 1.6.2013. [Online]. www.sggg.ch/de/members news/1005. [Zugriff am 20.2.2015].

2 Bayer. Yasmin-Fachinformation. Swissmedic, 11.9.2012. [Online]. www.swissmedicinfo.ch. [Zugriff am 2.2.2015].

3 Bayer. Yasmin-Patienteninformation. Basel: Documed AG; 2004

4 BGer, 4A 365/2014.

5 Bayer. Yasmin-Fachinformation. Basel: Documed AG; 2007.

6 Bayer. Yasmin-Patienteninformation. Swissmedic. 11.9.2012. [Online]. www.swissmedicinfo.ch. [Zugriff am 2.2.2015]

7 Beck K.Krankenversicherer und systematische Behandlungsfehler - Das Fallbeispiel Yasmin. In Mathwig F, Meireis T, Porz R (Eds.). «Fehlerfreundlichkeit» und Nichtschadensprinzip. Zürich: TVZ; 2015. Erscheint demnächst.

8 Gigerenzer G, Muir GJ. Launching the Century of the Patient. In Gigerenzer G, Muir GJ (Eds.). Better Doctors, Better Patients, Better Decisions. Frankfurt am Main: Strüngmann Forum Reports; 2009. S.3-28.

9 James AH. Venous Thromboembolism in Pregnancy. Arterioscler Thromb Vasc Biol. 2009;29:326-31.

10 Arzneimittelinformation. Thromboembolierisiko drospirenonhaltiger Kontrazeptiva (Yasmin u.a.). arzneitelegramm. 2007;38(10):95-6.

11 Pund PM, Plante L. Venous Thromboembolic Disease and Pregnancy. The New England Journal of Medicine: 2008;(6):202533.

12 Bundesamt für Statistik, Statistisches Jahrbuch der Schweiz 2015. Zürich: NZZ. 2015.

13 Bundesamt für Statistik. Schwangerschaftsabbrüche in der Schweiz. [Online]. www.bfs.admin.ch/bfs/portal/de/index/ themen/14/02/03/key/03.html [Zugriff am 8.4.2015].

14 Swissmedic. Stellungnahme zum Manuskript von K. Beck. Naive Haftpflichtklage oder tragischer Regulierungsfehler (Version vom 23.6.15). Swissmedic. 2015 